\title{
Probabilistic Models of Set-Dependent and Attribute-Level Best-Worst Choice
}

\author{
A. A. J. Marley ${ }^{*, 1}$ Terry N. Flynn ${ }^{2}$ \\ and
}

J. J. Louviere ${ }^{3}$

January 1, 2008

*Corresponding author:

${ }^{1}$ A.A.J. Marley

${ }^{2}$ Terry N. Flynn
Department of Social Medicine
University of Bristol
Canynge Hall
Whiteladies Road
Bristol BS8 $2 \mathrm{PR}$
England
Phone: +44.(0)117.928.7375
FAX: +44.(0)117. 928.7325
email: terry.flynn@bristol.ac.uk

Department of Psychology

University of Victoria

PO Box 3050 STN CSC

Victoria BC V8W 3P5

Canada

${ }^{3}$ Jordan J. Louviere

School of Marketing

University of Technology Sydney

PO Box 123, Broadway

Sydney, NSW

Australia

Phone: +61.2.9514.3993

FAX: +61.2 .9514 .3535$

email: jordan.louviere@uts.edu.au 


\begin{abstract}
We characterize a class of probabilistic choice models where the choice probabilities depend on two scales, one with a value for each available option and the other with a value for the set of available options. Then, we develop similar results for a task in which a person is presented with a profile of attributes, each at a pre-specified level, and chooses the best or the best and the worst of those attribute-level combinations. The latter design is an important variant on previous designs using best-worst choice to elicit preference information, and there is various evidence that it yields reliable interpretable data. Nonetheless, the data from a single such task cannot yield separate measures of the "importance" of an attribute and the "utility" of an attribute-level. We discuss various empirical designs, involving more than one task of the above general type, that may allow such separation of importance and utility.
\end{abstract}

keywords: attribute choice, best-worst choice, importance, probabilistic choice, profile 


\section{Introduction}

Over the past decade or so, a choice task in which a person is asked to select both the best and the worst option in an available set of options has seen increased applications over more traditional choice tasks, such as asking a person to choose a) the best option; b) choose the worst option; c) rank the options; or d) rate the options. Marley and Louviere (2005) summarized that work and developed an integrative theoretical approach to three overlapping classes of probabilistic models for best, worst, and best-worst choices, with the models in each class proposing specific ways in which such choices might be related.

In this paper, we discuss a different task, and associated experimental de$\operatorname{sign}(\mathrm{s})$, that involves several profiles of attributes, with the levels of the attributes varying between profiles. This type of task is commonly referred to as a "conjoint task" (e.g., Louviere 1988). When the profiles are generated by a suitable experimental design, such as a factorial design or an orthogonal fractional factorial design, one can view each profile generated in this way as a choice set of attribute levels ${ }^{1}$. When each profile is viewed as a choice set, and if a person is asked to simultaneously choose the best and the worst attribute-level (most and least attractive level or the attribute-level that, respectively, matters most and least) in each profile, the task parallels that considered in Marley and Louviere (2005). However, it differs empirically and theoretically in that in the profile case each choice set is constrained to contain an attribute-level for each attribute, whereas in the previously studied best-worst task, any combination of choice options can occur as a choice set.

Although this type of choice task may sound unusual, evidence exists that it yields reliable, interpretable data if one constructs the profiles using a suitable experimental design (see Coast et al., 2006; Louviere 1994; McIntosh \& Lou-

\footnotetext{
${ }^{1}$ In the remainder of the paper, we use "attribute-level" for a specific level of an attributte.
} 
viere, 2002). We demonstrate that choice data from a single such task cannot meaningfully separate the "importance" of each attribute and the "utility" associated with each level of an attribute. Towards the end of the paper, we [ajm: summarize prior research on separating "weights" and "values" and then discuss various discrete choice tasks] that may allow one to separate importance and utility if repeated for different substantive choice contexts. The general issues that arise are similar, but not identical, to those that arise when one studies the identifiability of scale factors (vis, variance parameters) in underlying random utility representations (see Louviere, Hensher \& Swait, 2003, Chapters 8 and 13; Swait \& Louviere, 1993).

Section 2 provides an illustration of the type of design that interests us in this paper. Section 3 characterizes a class of probabilistic choice models where the choice probabilities depend on two scales, one with a value for each available option and the other with a value for the set of available options. These characterizations guide us in the development in Section 4 of parallel models where each option is specified in terms of a vector of attribute-levels. We study the special case where a person has to select the best (and, possibly, the worst) attribute-level of the presented option. Section 5 presents special cases of these general models where the impact of each attribute-level is determined by a product of an importance weight for the attribute and a utility value for the specific level on that attribute. [ajm: Section 6 summarizes prior research on separating "weights" and "values", followed by a discussion of how one might achieve such a separation using discrete choice tasks.]

Finally, we note that the notation and derivations are quite complex, but we believe that they are necessary to reach the suggestions in Section 6.2 and the conclusions in Section 7 regarding issues surrounding the measurement of "importance." 


\section{An Illustration of Best-Worst Attribute-Level Choice}

Coast et al (2006) present a study of patients' preferences for attributes of care using best-worst attribute-level choice (the later is discussed below and defined formally in Section 4.2). The context is the fact that, in the UK, general practitioners with additional training in a specialist service are being used increasingly to provide service in a primary, rather than a hospital, setting. Coast et al.'s discrete choice experiment (DCE) involved varying levels of attributes of dermatology care that were found to be important to patients (Table 1). The attributes and levels were combined using a fractional factorial design to construct 16 scenarios; the scenarios were presented to each of 60 participants who provided complete data. The design used in the study enabled all main effects to be estimated and would have allowed all two-way interactions to be estimated had the utility of waiting time been linear in real time, which it was not. Table 2 shows a typical scenario, with a participant instructed to mark the "best thing" and the "worst thing" in that scenario, plus say whether or not they would attend an appointment that exhibited this combination of attribute-levels. The choice data were analyzed using the attribute-level maxdiff model presented in Section 4.2 .

Using the term impact in the sense of "influence on the choices made" (discussed in more detail in Sections 4 and 5), and assuming that the attribute-level maxdiff model (Section 4.2) holds, a researcher can measure ${ }^{2}$ the impact of each attribute on the choices (Table 3) as well as the impact of each attribute-level on the choices (Figure 1). In particular, the estimated impact of an attribute in Table 3 is the average of the impacts for the attribute-levels of that attribute in

\footnotetext{
${ }^{2}$ These measurements are on a common ratio scale (see Section 4.2), with the results in Tables 3 and 4 based on the log of that scale. This scale restriction is an important property of the attribute-level maxdiff model.
} 
Figure 1. Note that in this example the attribute "expertise" of the doctor has the most impact and the attribute "time waited" the least impact (Table 3). Also note that attribute-levels "thorough" and "expert 5 yrs" each have a large impact, while "not thorough" has little (least) impact (Figure 1) ${ }^{3}$. Please note that we have been careful to use the term "impact" rather than "importance" even though these terms might seem synonymous. The distinction is important for our work in this paper, and the required distinction has a long, tortuous history in psychological research, [ajm: some of which we summarize in Section 6.1]. For instance, Shanteau (1980) says "Thus, the impact that a variable has on a judgment can show up either in the weight or the scaling value (or both)" (p. 6) and "There is, as yet, no known procedure that can provide . . . an uncontaminated estimate of weight" (p. 33). Similarly, Flynn, Louviere, Peters and Coast (2006) say "Statements like 'quality of care is more highly valued than waiting time' can neither be supported nor refuted by comparisons of utility parameters from a traditional discrete choice experiment (DCE)" (Abstract).

In summary: An important goal of this paper is to contribute to our understanding of how we might measure separately the importance and the utility of each level of an attribute using discrete choice experiments. Achieving such understanding requires the clear formulation of the mathematical properties of attribute based models of best, worst and best-worst choice. We approach such understanding by, first, characterizing models for standard choice tasks where a decision maker chooses one option from a set of available options, such as medical appointments with different characteristics. Then, we adapt the notation and results to choices from a set of attribute-levels, as exemplified by the above medical appointments example.

\footnotetext{
${ }^{3}$ Coast et al (2006) restrict the term "impact" to the average of the scale values on an attribute and use the term "utility" for the scale value of an attribute at a specific level. We believe that "impact" is the appropriate term for both contexts.
} 


\section{Set-Dependent Choice Models}

Let $T$, with $|T| \geq 2$, denote the finite set of potentially available choice options, and for any subset $X \subseteq T$, with $|X| \geq 2$, let $B_{X}(x)$ denote the probability that the alternative $x$ is chosen as best in $X, W_{X}(y)$ the probability that the alternative $y$ is chosen as worst in $X$, and $B W_{X}(x, y)$ the probability that, jointly, the alternative $x$ is chosen as best in $X$ and the alternative $y \neq x$ is chosen as worst in $X$. Thus

$$
0 \leq B_{X}(x), W_{X}(y), B W_{X}(x, y) \leq 1
$$

and

$$
\sum_{x \in X} B_{X}(x)=\sum_{y \in X} W_{X}(y)=\sum_{\substack{x, y \in X \\ x \neq y}} B W_{X}(x, y)=1 .
$$

For simplicity, we assume that no choice probability equals 0 or 1 . Extensions to the general case can be made as in Luce (1959/2005).

Given $T$ and a particular set $X, X \subseteq T$, we refer to the set $\left\{B_{X}(x), x \in X\right\}$, $\left\{W_{X}(y), y \in X\right\},\left\{B W_{X}(x, y), x, y \in X, x \neq y\right\}$, respectively, as a set of best, worst, best-worst choice probabilities (on $X$ ). We have a complete set of best, worst, best-worst choice probabilities, respectively, (on a master set $T$ ) when we have a set of best, worst, best-worst choice probabilities on each $X, X \subseteq T$. Unless stated otherwise, in the basic theoretical developments we assume that we have a complete set of best, worst, best-worst choice probabilities, respectively, on a finite master set $T$ with $|T| \geq 2$.

In fact, we concentrate on models for best and best-worst choices, with those for worst choices being exactly parallel. Although we focus on complete sets of choice probabilities, in practice many applications study a sample of the complete set of probabilities. Typically, the sample is based on an appropriate 
experimental design for testing various models, such as a $2^{n}$ fractional factorial, which ensures that each option, and each pair of distinct options, is presented equally often across the selected subsets of average size $n / 2$ of the master set (Finn and Louviere, 1992). Similarly, designs known as balanced incomplete block designs (e.g., Street \& Street, 1987) are used often to construct choice sets, all of equal size, so as to ensure that all options appear equally often across all choice sets, and each pair of distinct options also jointly occur equally often across all choice sets. Such designs do not present all possible subsets of the master set $T$, i.e., they do not provide a complete set of choice frequencies (probabilities) on $T$, and so further theoretical work is needed to relate the present results to the properties of such designs. Also, the design issues are somewhat more complex for our main focus here, namely best-worst attributelevel choice, but are, nonetheless, well-understood (see Louviere \& Woodworth, 1983; Louviere, 1988; and Louviere, Hensher \& Swait 2003).

\subsection{Set-dependent Luce model}

We begin with models for best choices, then consider their extension to bestworst choices. Exactly parallel results can be developed for worst choices.

Definition 1 A complete set of best choice probabilities on a finite set $T$ satisfies a set-dependent Luce model iff there exist a positive scale $b$ on $T$ and a positive scale $\varphi$ on the subsets of $T$ with two or more elements such that for every $x \in X \subseteq T,|X| \geq 2$,

$$
B_{X}(x)=\frac{b(x)^{\varphi(X)}}{\sum_{z \in X} b(z)^{\varphi(X)}} .
$$

It satisfies Luce's model iff $\varphi(X)=1$ for every $X \subseteq T,|X| \geq 2$. 
Note that if (1) holds with $\varphi(X)=c, c>0$, for every $X \subseteq T,|X| \geq 2$, then if we define the positive scale $b^{\prime}$ by $b^{\prime}=b^{\varphi(X)}$, then (1) holds with $b$ replaced by $b^{\prime}$ and $\varphi(X)=1$ for every $X \subseteq T,|X| \geq 2$. Thus, no generality is lost in the way that we have defined Luce's model, which, in fact, agrees with the standard representation of that model.

In the following, we use the concepts of a log-interval and a ratio scale: $\mathrm{A}$ scale is a log-interval scale if the admissible transforms are functions of the form $\alpha x^{\beta}, \alpha, \beta>0$ and a ratio scale if the admissible transforms are functions of the form $\gamma x, \gamma>0$. We say that a ratio scale has a unit that is the reciprocal of that of a log-interval scale when the admissible transforms are linked via $\gamma=1 / \beta$.

Now, we show that the following condition characterizes the set-dependent Luce model, Def. 1.

Definition 2 A complete set of best choice probabilities on a finite set $T$ satisfies the set-dependent constant ratio rule iff for all $X \subseteq T, Y \subseteq T$, $|X| \geq 2,|Y| \geq 2, r, s, u, v \subseteq X \cap Y$,

$$
\frac{\log \frac{B_{X}(r)}{B_{X}(s)}}{\log \frac{B_{Y}(r)}{B_{Y}(s)}}=\frac{\log \frac{B_{X}(u)}{B_{X}(v)}}{\log \frac{B_{Y}(u)}{B_{Y}(v)}} .
$$

It satisfies the constant ratio rule iff each of the (overall) ratios in (2) equals 1.

Theorem 3 A complete set of best choice probabilities on a finite set $T$ satisfies a set-dependent Luce model, Def. 1, iff it satisfies the set-dependent constant ratio rule, (2). The scale $b$ is a log-interval scale and the scale $\varphi$ is a ratio scale with unit the reciprocal of that of $b$. The set satisfies Luce's model iff it satisfies the constant ratio rule.

All proofs are in Section 8. 


\section{$3.2 \quad$ Set-dependent maxdiff model}

Now, we present an extension of the set-dependent Luce model (for best choices) to best-worst choices.

Definition 4 A complete set of best-worst choice probabilities on a finite set $T$ satisfies a set-dependent maxdiff model iff there exist a positive scale $b$ on $T$ and a positive scale $\phi$ on the subsets of $T$ with two or more elements such that for every $x, y \in X \subseteq T, x \neq y,|X| \geq 2$,

$$
B W_{X}(x, y)=\frac{[b(x) / b(y)]^{\phi(X)}}{\sum_{\substack{\mathbf{r}, \mathbf{s} \in X \\ \mathbf{r} \neq \mathbf{s}}}[b(r) / b(s)]^{\phi(X)}} \quad(x \neq y) .
$$

It satisfies the maxdiff model iff $\phi(X)=1$ for every $X \subseteq T,|X| \geq 2$.

Note that if (3) holds with $\phi(X)=c, c>0$, for every $X \subseteq T,|X| \geq 2$, then if we define the positive scale $b^{\prime}$ by $b^{\prime}=b^{\phi(X)}$, then (3) holds with $b$ replaced by $b^{\prime}$ and $\phi(X)=1$ for every $X \subseteq T,|X| \geq 2$. Thus, no generality is lost in the way that we have defined the maxdiff model, which, in fact, agrees with the standard representation of that model as given in Marley \& Louviere (2005).

We now state conditions that characterize the set-dependent maxdiff model.

Definition 5 A complete set of best-worst choice probabilities on a finite set $T$ satisfies the set-dependent best-worst constant ratio rule iff for all $X \subseteq T$, $Y \subseteq T,|X| \geq 2,|Y| \geq 2, r, s, u, v \in X \cap Y$,

$$
\frac{\log \frac{B W_{X}(r, s)}{B W_{X}(s, r)}}{\log \frac{B W_{Y}(r, s)}{B W_{Y}(s, r)}}=\frac{\log \frac{B W_{X}(u, v)}{B W_{X}(v, u)}}{\log \frac{B W_{Y}(u, v)}{B W_{Y}(v, u)}}
$$

It satisfies the best-worst constant ratio rule iff the each of (overall) ratios in (4) equals 1. 
Definition 6 A complete set of best-worst choice probabilities on a finite set $T$, $|T| \geq 2$, satisfies 2 -invertibility iff for all $X \subseteq T,|X| \geq 2$, and $r, s, u, v \in X$, $r \neq s, u \neq v$,

$$
B W_{X}(r, s) B W_{X}(s, r)=B W_{X}(u, v) B W_{X}(v, u) .
$$

Definition 7 A complete set of best-worst choice probabilities on a finite set $T,|T| \geq 3$, satisfies 3 -reversibility iff for all $X \subseteq T,|X| \geq 3$, and distinct $r, s, t \in X, r \neq s, s \neq t, t \neq r$

$$
B W_{X}(r, s) B W_{X}(s, t) B W_{X}(t, r)=B W_{X}(r, t) B W_{X}(t, s) B W_{X}(s, r) .
$$

Note that constraints such as $r \neq s$ in (5) could be omitted as they are implicit in the fact that $r$ is best and $s$ is worst. However, we retain such constraints throughout the paper as it is necessary to ensure that they hold in various constructions that arise in the proofs.

For simplicity, in the statement of the following theorem we assume that 3 -reversibility holds vacuously when $|T|=2$.

Theorem 8 A complete set of best-worst choice probabilities on a finite set $T,|T| \geq 2$, satisfies a set-dependent maxdiff model, Def. 4, iff it satisfies the set-dependent best-worst constant ratio rule, (4), 2-invertibility, (5), and 3-reversibility, (6). The scale $b$ is a loginterval scale and the scale $\phi$ is a ratio scale with unit the reciprocal of that of $b$. The set satisfies the maxdiff model iff it satisfies the best-worst constant ratio rule, 2-invertibility, (5), and 3-reversibility, (6) in which case b is a ratio scale. 


\section{Probabilistic Models of Best and Best-Worst Choice for Attribute-Level Profiles}

We now develop models for a rather different type of task. However, the results are stated and proved in a manner analogous to those already presented.

For clarity, we first present notation and models for best choices between a set of options, each of which is specified in terms of a vector of attribute-levels. This task parallels that presented in the previous section. Then, we turn to our main focus, namely, best-worst choice between a set of attribute-levels.

We assume the following: there are $m \geq 2$ attributes, and we let $M=$ $\{1, \ldots, m\}$. Attribute $i, i=1, \ldots, m$, has $q(i)$ levels, and we let $Q(i)=\{1, \ldots, q(i)\}$ and $Q=\prod_{i=1}^{m} Q(i)$. A profile is an $M$-component vector with each component $i$ taking on one of the $q(i)$ levels for that component. Thus we have $Q$ possible profiles. We denote a typical profile by

$$
\mathbf{z}=\left(z_{1}, \ldots, z_{m}\right)
$$

where $z_{i}, i=1, . ., m$, denotes the level of attribute $i$ in profile $\mathbf{z}$. As previously, we call each such $z_{i}$ an attribute-level ${ }^{4}$.

Thus, $Q$, with $|Q| \geq 2$, is the finite set of potentially available profiles, i.e., in (7), $\mathbf{z} \in Q$. We let $X \subseteq Q,|X|=n \geq 2$, denote a typical subset of these profiles. Proceeding as earlier, with $\mathbf{x}, \mathbf{y} \in X$, let $B_{X}(\mathbf{x})$ denote the probability that the profile $\mathbf{x}$ is chosen as best in $X, W_{X}(\mathbf{y})$ the probability that the profile $\mathbf{y}$ is chosen as worst in $X$, and $B W_{X}(\mathbf{x}, \mathbf{y})$ the probability that, jointly, the profile $\mathbf{x}$ is chosen as best in $X$ and the profile $\mathbf{y} \neq \mathbf{x}$ is chosen as worst in $X$.

\footnotetext{
${ }^{4}$ A more complete notation would have extra subscripts, e.g., $\mathbf{r}_{\mathbf{z}}=\left(r_{1, z_{1}}, \ldots, r_{m, z_{m}}\right)$, where $\mathbf{z}=\left(z_{1}, \ldots, z_{m}\right)$ is the vector of attribute-levels. We do not believe that any confusion arises with the simpler notation.
} 
Thus

$$
0 \leq B_{X}(\mathbf{x}), W_{X}(\mathbf{y}), B W_{X}(\mathbf{x}, \mathbf{y}) \leq 1
$$

and

$$
\sum_{\mathbf{x} \in X} B_{X}(\mathbf{x})=\sum_{\mathbf{y} \in X} W_{X}(\mathbf{y})=\sum_{\substack{\mathbf{x}, \mathbf{y} \in X \\ \mathbf{x} \neq \mathbf{y}}} B W_{X}(\mathbf{x}, \mathbf{y})=1 .
$$

For simplicity, we assume that no choice probability equals 0 or 1 . Extensions to the general case can be made as in Luce (1959/2005).

Now, we can develop models for a complete set of best, worst, best-worst probabilities for choices from such profiles exactly paralleling those introduced above for choices from generic options. We do not do so because, without further assumptions about the representation of profiles, such results add nothing to those we have already presented. Instead, in the following section, we consider the case where at each choice opportunity the choice set consists of a single profile, say $\mathbf{z}=\left(z_{1}, \ldots, z_{m}\right)$, selected from a set, $P$, of possible profiles, and the participant has to indicate the best and the worst attribute-level in that profile. The available empirical results indicate that participants understand the task and give reliable data (Coast et al., 2006; Louviere, 1994). The notation then is: for a typical profile $\mathbf{z} \in P$ and $i \in M, B W_{\mathbf{z}}\left(z_{i}\right)$ is the probability that the attribute-level $z_{i}$ is chosen as best in $\mathbf{z}$, and for $i, j \in M, i \neq j, B W_{\mathbf{z}}\left(z_{i}, z_{j}\right)$ is the probability that, jointly, the attribute-level $z_{i}$ is chosen as best in $\mathbf{z}$ and the attribute-level $z_{j}$ is chosen as worst in z. Also,

$$
0 \leq B W_{\mathbf{z}}\left(z_{i}\right), B W_{\mathbf{z}}\left(z_{i}, z_{j}\right) \leq 1
$$

and

$$
\sum_{i \in M} B W_{\mathbf{z}}\left(z_{i}\right)=\sum_{\substack{i, j \in M \\ i \neq j}} B W_{\mathbf{z}}\left(z_{i}, z_{j}\right)=1
$$




\subsection{Attribute-level Luce model}

Now, we develop models for choices between attribute-levels, first for best choices to illustrate the theoretical approach, then for best-worst choice, the case of real interest to us.

Definition 9 A set of best choice probabilities ${ }^{5}$ on a finite set, $P$, of profiles satisfies an attribute-level Luce model iff there exists a positive scale b on the attributes such that for every profile $\mathbf{z} \in P$ with attribute-levels $z_{i}, i \in M$,

$$
B_{\mathbf{z}}\left(z_{i}\right)=\frac{b\left(z_{i}\right)}{\sum_{j \in M} b\left(z_{j}\right)}
$$

The structure of the conditions that characterize this model are similar in form to those for the set-dependent maxdiff model of Section 3.2. Nonetheless, we restate them here as the notation, and thus the proofs, differ.

It is likely useful when studying the following definition to consult the summary notation following the definition. Also, an intuitive understanding of the conditions in the definition can be obtained by noting that when the attributelevel Luce model, (8), holds, then, for each equation in the following definition, the product of the terms in the numerator (respectively, denominator) on the left- and right-hand-sides are equal, and so the conditions hold for that model. This observation applies throughout the remainder of the paper. Also, in the following definition and later related ones, we use "invertibility" and "reversibility" for properties similar to those of Defs. 6 and 7. No confusion should arise from this duplicate usage.

Definition $10 A$ set of best choice probabilities on a finite set of profiles $P$

\footnotetext{
${ }^{5}$ A more complete phrasing would be "best attribute-level choice probabilities." We do not think the more compact phrasing leads to confusion here, or in parallel later definitions.
} 
satisfies 2-reversibility iff for all profiles $\mathbf{r}, \mathbf{s}$ with $r_{i}=s_{i}, s_{j}=r_{j}, i \neq j$,

$$
B_{\mathbf{r}}\left(r_{i}\right) B_{\mathbf{s}}\left(s_{j}\right)=B_{\mathbf{r}}\left(r_{j}\right) B_{\mathbf{s}}\left(s_{i}\right)
$$

It satisfies 3-reversibility iff for all profiles $\mathbf{r}, \mathbf{s}, \mathbf{t}$ with $r_{i}=t_{i}, s_{j}=r_{j}$, $t_{k}=s_{k}, i \neq j, j \neq k$,

$$
B_{\mathbf{r}}\left(r_{i}\right) B_{\mathbf{s}}\left(s_{j}\right) B_{\mathbf{t}}\left(t_{k}\right)=B_{\mathbf{r}}\left(r_{j}\right) B_{\mathbf{s}}\left(s_{k}\right) B_{\mathbf{t}}\left(t_{i}\right)
$$

It satisfies 4-reversibility iff for all profiles $\mathbf{r}, \mathbf{s}, \mathbf{t}, \mathbf{u}$, with $r_{i}=u_{i}, s_{j}=r_{j}$, $t_{k}=s_{k}, u_{l}=t_{l}, i \neq j, j \neq k, k \neq l$

$$
B_{\mathbf{r}}\left(r_{i}\right) B_{\mathbf{s}}\left(s_{j}\right) B_{\mathbf{t}}\left(t_{k}\right) B_{\mathbf{u}}\left(u_{l}\right)=B_{\mathbf{r}}\left(r_{j}\right) B_{\mathbf{s}}\left(s_{k}\right) B_{\mathbf{t}}\left(t_{l}\right) B_{\mathbf{u}}\left(u_{i}\right)
$$

Note that 4 -reversibility includes $3-$ and 2 -reversibility as special cases: first, take $l=i$ to get 3 -reversibility, then add $i=k$ to get 2 -reversibility. Nonetheless, we retain $i$-reversibility, $i=2,3,4$, as separate conditions for clarity in the proof of the following theorem.

Also, the form of 2 -reversibility is more transparent if we let $\bullet_{i}=r_{i}=s_{i}$, $\bullet_{j}=r_{j}=s_{j}$, which reduces it to

$$
B_{\mathbf{r}}\left(\bullet_{i}\right) B_{\mathbf{s}}\left(\bullet_{j}\right)=B_{\mathbf{r}}\left(\bullet_{j}\right) B_{\mathbf{s}}\left(\bullet_{i}\right)
$$

and, with $\bullet_{i}=r_{i}=t_{i}, \bullet_{j}=r_{j}=s_{j}=, \bullet_{k}=s_{k}=t_{k}, 3$-reversibility reduces to

$$
B_{\mathbf{r}}\left(\bullet_{i}\right) B_{\mathbf{s}}\left(\bullet_{j}\right) B_{\mathbf{t}}\left(\bullet_{k}\right)=B_{\mathbf{r}}\left(\bullet_{j}\right) B_{\mathbf{s}}\left(\bullet_{k}\right) B_{\mathbf{t}}\left(\bullet_{i}\right),
$$

with a similar notational version of 4 -reversibility. However, we retain the detailed versions so that the proof of the following theorem is clear. 
Theorem $11 A$ set of best choice probabilities on a finite set of profiles $P$ satisfies an attribute-level Luce model, Def. 9, with b a ratio scale, iff it satisfies 2-, 3- and 4-reversibility, Def. 10.

Next, we define impact, which we used informally in Section 2 as meaning "influence on the choices made," and which is important for our later discussion of how one might separate the contributions of the "importance" of an attribute from the "utility" of an attribute-level: Given an attribute-level Luce model, Def. 9, with $b$ a ratio scale, then, for each attribute $i \in M$ and each attributelevel $z_{i}, b\left(z_{i}\right)$ is the impact of the attribute-level $z_{i}$, and the (arithmetic or geometric) average of $b\left(z_{i}\right)$ over the attribute-levels $z_{i}$ for attribute $i$ is the (average) impact of the attribute $i$. We can estimate the scale values $b\left(z_{i}\right)$, i.e., the impacts, from data, and, importantly, they are all measured on a common ratio scale. To our knowledge, this is the first demonstration of a model, and an associated experimental procedure, that allows such measurement of attributelevels on a common scale across attributes.

\subsection{Attribute-level maxdiff model}

Now we extend the definitions and results for best (attribute-level) choices to best-worst (attribute-level) choices.

Definition 12 A set of best-worst choice probabilities on a finite set of profiles $P$ satisfies an attribute-level maxdiff model iff there exist a positive scale $b$ on the attributes such that for every profile $\mathbf{z} \in P$ and $i, j \in M, i \neq j$,

$$
B W_{\mathbf{z}}\left(z_{i}, z_{j}\right)=\frac{b\left(z_{i}\right) / b\left(z_{j}\right)}{\sum_{\substack{k, l \in M \\ k \neq l}}\left[b\left(z_{k}\right) / b\left(z_{l}\right)\right]} \quad(i \neq j) .
$$

The structure of the conditions that characterize this model parallels those for the set-dependent maxdiff model in Section 3.2. Nonetheless, the notation, 
and thus the proofs, differ. As in that case, it is likely useful when studying the following definitions to consult the summary notation introduced following the definitions. Also, as stated previously, an intuitive understanding of the conditions in the definition can be obtained by noting that when the attributelevel maxdiff model, (12), holds, then, for each equation in the definition, the product of the terms in its numerator (respectively, denominator) on the leftand right-hand-sides are equal, and so the conditions hold for that model.

Definition 13 A set of best-worst choice probabilities on a finite set of profiles $P$ satisfies 2-invertibility iff for every profile $\mathbf{r} \in P$, and attribute-levels $r_{i}, r_{j}$, $r_{k}, r_{l}, i, j, k, l \in M, i \neq j, k \neq l$

$$
B W_{\mathbf{r}}\left(r_{i}, r_{j}\right) B W_{\mathbf{r}}\left(r_{j}, r_{i}\right)=B W_{\mathbf{r}}\left(r_{k}, r_{l}\right) B W_{\mathbf{r}}\left(r_{l}, r_{k}\right)
$$

Definition 14 A set of best-worst choice probabilities on a finite set of profiles $P$ satisfies 3-reversibility iff for all profiles $\mathbf{r}, \mathbf{s}, \mathbf{t}$ with $r_{j}=s_{j}, s_{k}=t_{k}, t_{i}=r_{i}$, $i \neq j, j \neq k$,

$$
\begin{aligned}
& B W_{\mathbf{r}}\left(r_{i}, r_{j}\right) B W_{\mathbf{s}}\left(s_{j}, s_{k}\right) B W_{\mathbf{t}}\left(t_{k}, t_{i}\right) \\
& \quad=B W_{\mathbf{t}}\left(t_{i}, t_{k}\right) B W_{\mathbf{s}}\left(s_{k}, s_{j}\right) B W_{\mathbf{r}}\left(r_{j}, r_{i}\right) .
\end{aligned}
$$

It satisfies 4-reversibility iff for all profiles $\mathbf{r}, \mathbf{s}, \mathbf{t}, \mathbf{u}$, with $, r_{j}=s_{j}, s_{k}=t_{k}$, $t_{l}=u_{l}, u_{i}=r_{i}, i \neq j, j \neq k, k \neq l$

$$
\begin{aligned}
& B W_{\mathbf{r}}\left(r_{i}, r_{j}\right) B W_{\mathbf{s}}\left(s_{j}, s_{k}\right) B W_{\mathbf{t}}\left(t_{k}, t_{l}\right) B W_{\mathbf{u}}\left(u_{l}, u_{i}\right) \\
& \quad=B W_{\mathbf{u}}\left(u_{i}, u_{l}\right) B W_{\mathbf{t}}\left(t_{l}, t_{k}\right) B W_{\mathbf{s}}\left(s_{k}, s_{j}\right) B W_{\mathbf{r}}\left(r_{j}, r_{i}\right) .
\end{aligned}
$$

Note that we cannot obtain 3-reversibility as a special case of 4-reversibility by setting $i=l$ because $B W_{\mathbf{u}}\left(u_{i}, u_{i}\right)$ is undefined, or, conceptually, it equals 0 . 
Also, the form of 3 -reversibility reduces to

$$
\begin{aligned}
& B W_{\mathbf{r}}\left(\bullet_{i}, \bullet_{j}\right) B W_{\mathbf{s}}\left(\bullet_{j}, \bullet_{k}\right) B W_{\mathbf{t}}\left(\bullet_{k}, \bullet_{i}\right) \\
& \quad=B W_{\mathbf{t}}\left(\bullet_{i}, \bullet_{k},\right) B W_{\mathbf{s}}\left(\bullet_{k}, \bullet_{j}\right) B W_{\mathbf{r}}\left(\bullet_{j}, \bullet_{i}\right),
\end{aligned}
$$

with a similar notational version of 4 -reversibility.

However, we retain the detailed versions so that the proof to the following theorem is clear.

Theorem 15 A set of best-worst choice probabilities on a finite set of profiles $P$ satisfies an attribute-level maxdiff model, Def. 12, with $b$ a ratio scale iff it satisfies 2-invertibility, Def 13, and 3-and 4-reversibility, Def. 14.

Given an attribute-level maxdiff model, Def. 12, with $b$ a ratio scale, then, using the terms as for the attribute-level Luce model, Def. 9, for each attribute $i \in M$ and each attribute-level $z_{i}$, we can estimate the impact $b\left(z_{i}\right)$ of the attribute-level $z_{i}$, and calculate the (arithmetic or geometric) average of $b\left(z_{i}\right)$ over the attribute-levels $z_{i}$ for attribute $i$, which is the (average) impact of attribute $i$. This is exactly the terminology and interpretation of the data presented in the dermatology example ${ }^{6}$ in Section 2.

\section{Weight Estimation in Attribute-Level Models}

Now, we turn to identifiability issues in models for the selection of the best attribute-level in a profile of attribute-levels when we assume that a typical attribute-level scale value $b\left(z_{i}\right)$ is a function of the "importance" of attribute $i$ and the "utility" of level $z_{i}$ on attribute $i$. We explore various ideas concerning how they may be made identifiable, obtaining very useful direction from the

\footnotetext{
${ }^{6}$ As noted earlier, Tables 3 and 4 use the values of $\log b\left(z_{i}\right)$ and their arithmetic mean.
} 
recent related work of Louviere et al (2002), Louviere (2004) and Louviere and Islam (2007). Similar issues arise in the separation of importance and utility in best-worst attribute-level choice models, which we illustrate briefly with the approach that appears to have the most potential.

We begin with what we believe is the appropriate extension of the attributelevel Luce model (for best choices).

\subsection{Weighted Attribute-Level Luce Model}

Definition $16 A$ set of best choice probabilities on a finite set of profiles $P$ satisfies a weighted attribute-level Luce model iff there exist a positive scale $\widetilde{b}$ on the attributes and positive constants $\beta_{i}, i \in M$, such that for every profile $\mathbf{z} \in P$ and attribute-level $z_{i}, i \in M$,

$$
B W_{\mathbf{z}}\left(z_{i}\right)=\frac{\widetilde{b}\left(z_{i}\right)^{\beta_{i}}}{\sum_{j \in M} \widetilde{b}\left(z_{j}\right)^{\beta_{j}}} .
$$

It satisfies an attribute-level Luce model iff $\beta_{i}=1$ for every $i \in M$.

Note that, with

$$
b\left(z_{i}\right)=\widetilde{b}\left(z_{i}\right)^{\beta_{i}}
$$

(16) becomes

$$
B W_{\mathbf{z}}\left(z_{i}\right)=\frac{b\left(z_{i}\right)}{\sum_{j \in M} b\left(z_{j}\right)}
$$

i.e., an attribute-level Luce model, Def. 9. Thus, the "weights" $\beta_{i}$ and "utility" values $\widetilde{b}\left(z_{i}\right)$ are not separately identifiable from a single design involving a finite set of profiles ${ }^{7}$. Nonetheless, for each such design, and for each $i \in M$, we can estimate the impact $b\left(z_{i}\right)$ of each attribute-level $z_{i}$, and, thus, also, the average impact of attribute $i$.

\footnotetext{
${ }^{7}$ We call the $\beta_{i}$ weights, rather than exponents, becuse if we let $u\left(r_{i}\right)=\log \widetilde{b}\left(r_{i}\right)$, then we obtain $b\left(r_{i}\right)=\exp \beta_{i} u\left(r_{i}\right)$. The latter form is the one that is often used when the model is derived as a random utility model (see Coast et al, 2006; Marley \& Louviere, 2005).
} 
Therefore, we explore possible designs for collecting data that may allow such a separation. However, first we define the corresponding version of the maxdiff model and show it has a parallel limitation.

\subsection{Weighted Attribute-Level Maxdiff Model}

Definition 17 A set of best-worst choice probabilities on a finite set of profiles $P$ satisfies a weighted attribute-level maxdiff model iff there exist a pos-

itive scale $\widetilde{b}$ on the attributes and positive constants $\beta_{i}, i \in M$, such that for every profile $\mathbf{z} \in P$ and attribute-levels $z_{i}, z_{j}, i, j \in M$,

$$
B W_{\mathbf{z}}\left(z_{i}, z_{j}\right)=\frac{\widetilde{b}\left(z_{i}\right)^{\beta_{i}} / \widetilde{b}\left(z_{j}\right)^{\beta_{j}}}{\sum_{\substack{k, l \in M \\ k \neq k}}\left[\widetilde{b}\left(z_{k}\right)^{\beta_{k}} / \widetilde{b}\left(z_{l}\right)^{\beta_{l}}\right]} \quad(i \neq j) .
$$

It satisfies an attribute-level maxdiff model iff $\beta_{i}=1$ for every $i \in M$.

Note that, with $b\left(z_{i}\right)=\widetilde{b}\left(z_{i}\right)^{\beta_{i}},(19)$ becomes

$$
B W_{\mathbf{z}}\left(z_{i}, z_{j}\right)=\frac{b\left(z_{i}\right) / b\left(z_{j}\right)}{\sum_{\substack{k, l \in M \\ k \neq l}}\left[b\left(z_{k}\right) / b\left(z_{l}\right)\right]} \quad(i \neq j),
$$

i.e., an attribute-level maxdiff model, Def. 12. Thus, as with the weighted attribute-level Luce model, the weights $\beta_{i}$ and utility values $\widetilde{b}\left(z_{i}\right)$ are not separately identifiable from a single design involving a finite set of profiles. Nonetheless, as in that case, for each such design, and for each $i \in M$, we can estimate the impact $b\left(z_{i}\right)$ of each attribute-level $z_{i}$, and, thus, also, the average impact of attribute $i$. 


\section{Separating Importance Weights and Utility Values}

[ajm: The following section is the new material, with some additional revisions marked [ajm:]

\subsection{Previous methods}

The problem of separating importance weights and scale values remains largely unresolved, despite considerable research. Two notable streams of [ajm: revised: relevant research stemmed from Anderson's (1970) information integration theory, namely, Norman's (1976a,b,c) method of relative information and Birnbaum's (1976 and later - see below) work related to the differentially weighted averaging model. Following Anderson's (1970) classic exposition of information integration theory, various papers examined conditions under which one can separate weight and scales. For example, Anderson (1973), in commenting on a paper by Schoenemann, Cafferty and Rotton (1973), acknowledged that [ajm: scale values must be equal across attributes - I do not understand that phrase - or Anderson's (1973) parallel statement "...the scale values, or at least their differences, are constant across position..." - "position" on what - each attribute?] for weights to be identified in the constant weight averaging model. This assumption was relaxed by [ajm: Please check that I have the correct cite: Norman (1976a)] where he systematically varied the presence/absence of attribute information to study differential weighting and noted also that non-additive integration processes would pose problems for his approach.]

Birnbaum (1976), Birnbaum and Wong (1976) and Birnbaum and Stegner (1979, 1981) describe experiments that tested additive, constant weight, and 
relative weight averaging models. Birnbaum and Zimmerman (1998) report a further series of experiments to test the differentially weighted averaging model and discriminate among other competing process models. Although these papers deal at least in part with the problem of separating weights and scales, they are only tangentially relevant to our best-worst choice work for the following reasons: [ajm: 1) Our focus is choice, in contrast to, say, rating. 2) the response method involved category rating scales or judgments on response scales that are assumed continuous or at least monotonically related to the latent dimension of interest; 3) the stimulus component involved numerical, continuous attributes where it is reasonable to assume that one can specify weights and/or scale values to be a function of the underlying physical dimension;][ajm: delete, or rephrase as we now admit this as a possible approach: 4) Birnbaum and Stegner and Birnbaum and Zimmerman focus on showing that context manipulations can be used to vary weights and scales; 5) Birnbaum and Stegner attempted to relate differences in weights to estimates of weights directly elicited from rating tasks; [ajm: however, ratings are subject to several criticisms (e.g., see Louviere and Islam 2008).]

Additionally, Luce (1981) [ajm: presents axioms that are sufficient to separate weight and scale in an averaging representation, noting] that "with an adequate amount of data, the weights of the averaging model are perfectly identifiable." What is relevant for our work on discrete choice processes applied to factorial and fractional factorial combinations of attribute levels is his further observation that "what is not yet clear is how to axiomatize averaging in the actual finite factorial designs usually used."

[ajm: added: Finally, there is one type of task where the separation of weights and values appears to be feasible, especially in choice tasks. This occurs when the "weights" and "values" are associated with separate variables that 
can be independently manipulated. An important such case is choice between gambles, where, in each gamble, each consequence (say, an amount of money) is associated with a separate event (say, a particular face occurring on the toss of a die). Birnbaum has empirical results in this domain that give support for his configural weighted utility model - see Marley and Luce (2005) for an integrative theoretical framework for the major contemporary theories in this domain, including the configural weighted utility model, and a summary of the status of these theories vis-a-vis data. Importantly, those models assume deterministic choice.]

[ajm: In summary, our work differs from that of Birnbaum and colleagues in that we focus on a specific type of "pick-any" discrete choice task in which individuals, at each choice opportunity, select the best/most attractive and worst/least attractive levels/cues from a profile of attributes levels. We do not see how to identify/separate weights and scales in the context of a single factorial or fractional factorial choice experiment of the type considered in this paper, in agreement with the final quote from Luce (1981), above. However, in the next section, we consider cases where one might also manipulate context or other relevant information that might change the way in which individuals respond to the choice task. In that sense, we agree with Birnbaum and his coauthors that manipulation of external factors such as decision context and/or varying/obtaining extra information may enable one to separate weights and scale values. Nonetheless, we conclude that the relevant discrete choice tasks are fraught with difficulty, paralleling those mentioned above for rating and other more classical methods.] 


\subsection{Possible methods using discrete choice experiments}

We discuss four approaches that give hope of separating importance weights and utility values in probabilistic models of best attribute-level choice and, by extension that we illustrate, in probabilistic models of best-worst attribute-level choice. Each approach requires considerable further theoretical and empirical study. Throughout, we assume that we have a set of profiles, with a typical example being that describing a possible trip with the $(m=$ six $)$ attributes being location, airline, duration, cost, travel time, date, and with an appropriate number of levels on each attribute. For simplicity, we begin with a discussion of best attribute-level choice, and later give an example with best-worst attributelevel choice.

\subsubsection{Approach 1}

Here, the experimental task involves presentation of a single profile including all the attributes at each choice opportunity, with each participant selecting the best attribute-level. We assume that each participant carries out the task under two different instructions, namely to consider the trip for business and for vacation $^{8}$. We assume that the weighted attribute-level Luce model, Def. 16, holds for each task, with a common utility scale $\widetilde{b}$ but possibly with different weights for each task. We show that, if the weights do differ (which is testable), then the scale values and weights may be separately identifiable.

The essential step is to recognize that it follows from (17) that the weights $\beta_{i}^{(\pi)}, \pi=1,2, i \in M$, for the two tasks, have to satisfy, for all $z_{i}$,

$$
\frac{\beta_{i}^{(1)}}{\beta_{i}^{(2)}}=\frac{\log b^{(1)}\left(z_{i}\right)}{\log b^{(2)}\left(z_{i}\right)},
$$

\footnotetext{
${ }^{8}$ Pretesting would be needed to decide whether or not both instructions could be mixed in a single design.
} 
where $b^{(\pi)}, \pi=1,2$, are the scale values in (8) that we can estimate using the theoretical results of Section 4.1, and data analysis methods that have been developed for this and related models (see Louviere et al, 2003). Then (21) requires that, for every $i \in M$ and all attribute-levels $r_{i}, s_{i}$,

$$
\frac{\log b^{(1)}\left(r_{i}\right)}{\log b^{(2)}\left(r_{i}\right)}=\frac{\log b^{(1)}\left(s_{i}\right)}{\log b^{(2)}\left(s_{i}\right)}
$$

The above is a necessary condition to be able to estimate the weights, which differ across the two contexts whenever the ratios in (22) are not all equal to 1. Although it does not add much, it is worth noting that (22) can be written in terms of observable data by substituting the scale values $b^{(\pi)}\left(z_{i}\right), \pi=1,2$, defined in (36) of Section 8, in (22).

Now, we require that each $\beta_{i}^{(\pi)} \geq 0, \pi=1,2, i \in M$, and, for simplicity, we assume that all the inequalities are strict, i.e., $\beta_{i}^{(\pi)}>0, \pi=1,2, i \in M$. These restrictions, combined with (22), yield the following necessary condition for separation of weights and utilities: for all $r_{i}, s_{i}, i \in M$,

$$
\frac{\log b^{(1)}\left(r_{i}\right)}{\log b^{(2)}\left(r_{i}\right)}=\frac{\log b^{(1)}\left(s_{i}\right)}{\log b^{(2)}\left(s_{i}\right)}>0
$$

However, we can now easily show that this necessary condition is also sufficient for the desired weights to exist. First, take a fixed $\mathbf{z}=\left(z_{1}, \ldots, z_{i}, \ldots, z_{m}\right)$, and, for $i \in M$, select arbitrary constants $\beta_{i}^{(2)}>0$ and then set

$$
\beta_{i}^{(1)}=\beta_{i}^{(2)} \frac{\log b^{(1)}\left(z_{i}\right)}{\log b^{(2)}\left(z_{i}\right)}
$$

Using these definitions and (23), it is clear that, as required, (21) and (22) hold for all attribute-levels $r_{i}, s_{i}, i \in M$. 
Finally, the scale values required in (19) are given by

$$
\widetilde{b}\left(z_{i}\right)=b^{(1)}\left(z_{i}\right)^{\frac{1}{\beta_{i}^{(1)}}}=b^{(2)}\left(z_{i}\right)^{\frac{1}{\beta_{i}^{(2)}}}
$$

with the equality of these two values following from (24).

However, it is customary to require that, for $\pi=1,2, \sum_{i=1}^{m} \beta_{i}^{(\pi)}=1$, and it is not obvious whether or not (23) is sufficient for weights to exist satisfying these constraints as well as (22) for all attribute-levels $r_{i}, s_{i}, i \in M$. In fact, if we restrict our attention to the case where each profile has two attributes, i.e., $m=2$, and there are two different instructions, then solving for the $\beta_{i}^{(\pi)}$ requires solving a nonlinear programming problem with the constraints: for $i, \pi=1,2$, $\sum_{i=1}^{m} \beta_{i}^{(\pi)}=1$ and $\beta_{i}^{(\pi)}>0$. We have derived the explicit solution, which exists only when there are specific relations between the values of

$$
\frac{\log b^{(1)}\left(r_{i}\right)}{\log b^{(2)}\left(r_{i}\right)}
$$

for $i=1,2$. It is not obvious how to derive the corresponding constraints for general values of $m$ and this may require the use of general nonlinear programming methods.

Thus, an open problem is to characterize the (best) weighted attribute-level Luce model.

Of course - and this is true of all the approaches that we discuss in this section - we could assume that the relevant theoretical assumptions are valid, in particular that the importance weights (possibly) vary across tasks and the utility values remain the same across tasks, and find the best fit to the data under these assumptions. However, without a clear understanding of the theoretical properties underlying the data, it may be difficult to reach clear conclusions about the adequacy of various fits to the data. 
A parallel open problem and issues apply to the weighted attribute-level maxdiff model.

\subsubsection{Approach 2}

Here, in addition to the tasks in Section 6.2.1, participants are asked to evaluate the six attributes using a constant sum scale (100 points) to indicate how important each attribute (not attribute-level) is relative to the other attributes in each task separately, i.e., under each of the two different instructions - business or pleasure (for an example with one task instruction, see Louviere \& Islam, 2007). We can now proceed, as in Section 6.2.1, to check whether or not those importance weights can be used, with utility values unchanged across the two tasks, to fit the (best) attribute-level Luce model to the best data under each of the two task instructions. Notice that we can carry out such fits in a standard way, such as maximum likelihood, since here we are assuming that the importance weights involved in each best attribute-level task are those obtained from the constant sum task. Thus, we do not need, though we will still like to have, a characterization of the attribute-level Luce model to test these ideas. Of course, if the importance weights obtained from the constant sum tasks are essentially the same for both tasks, then, as before, we cannot estimate the utility value for each attribute-level. Also, conceptually, this approach makes more sense in fitting the data of individual participants than in fitting group data, though it will be of interest to consider the latter and think more about when this type of approach might be valid for such fits.

[ajm: A variant of this approach involves an experimental design where subsets of the six attributes are presented for evaluation - see the related material in Section 6.2.4.] 


\subsubsection{Approach 3}

A variant on the approach used in Section 6.2.2 to obtain importance weights is to use best-worst designs where appropriately balanced subsets of the attributes (not attribute-levels) are presented to each participant who is instructed to select the most important and the least important attribute in each subset under each task instruction, i.e., business and pleasure (with one task instruction, see, for example, Finn \& Louviere, 1992; Louviere \& Islam, 2007). These bestworst attribute, not attribute-level, data are fit by the maxdiff model, with the resulting scale values being interpreted as the importance weights in the bestworst attribute-level tasks. One then proceeds as in Section 6.2.2, with parallel concerns.

It is important to note that, in this approach, it is assumed that the importance weights (may) depend on the task instruction (business versus pleasure) but do not depend on the currently presented subset of attributes. Next, we consider a related best-worst attribute task where we do assume that the importance weights (may) depend on the (sub)set of attributes presented.

\subsubsection{Approach 4}

Here, an experimental design is constructed that involves a set of partial profiles - that is, profiles where only a subset of the full attribute set is present in each profile. We assume that this set of partial profiles includes all attributes and all levels in a balanced manner across presentations - for instance, the attributes of the partial profiles can be selected according to a $2^{n}, n \leq m$, fractional factorial design. For each partial profile, a participant selects the best and the worst attribute-level of that profile (for a similar design for attributes, not attributelevels, see Louviere and Islam, 2007).

Now, assume that there is a (fixed) set of importance weights $\beta_{k}>0$, 
$k=1, \ldots, m$, with $\sum_{k=1}^{m} \beta_{k}=1$, that apply when, as in Section 6.2.1, a full profile is presented to the participant under a specific task instruction (e.g., vacation or business trip). Then a first assumption to consider is that the importance weights in a partial profile under the same task instruction are given by applying the Luce (MNL) choice model (Luce, 1959/2005) to these weights. To develop this assumption, for each partial profile $\Pi$, define the vector $a(\Pi)=\left(a_{1}(\Pi), \ldots, a_{m}(\Pi)\right)$ by

$$
a_{i}(\Pi)=\left\{\begin{array}{ccc}
1 & \text { if } & \text { attribute } i \text { occurs in partial profile } \Pi \\
0 & \text { otherwise }
\end{array},\right.
$$

and assume that, when partial profile $\Pi$ is presented, the importance weights, which may be called relative, are given by

$$
\beta_{i}(\Pi)=\frac{a_{i}(\Pi) \beta_{i}}{\sum_{j=1}^{m} a_{j}(\Pi) \beta_{j}} \quad(i \in M) .
$$

Note that the definition of the vector $a(\Pi)$ implies that $a_{k}(\Pi)=0$ for any attribute $k$ that is not present in the partial profile $\Pi$ and that the sum of the weights $\beta_{i}(\Pi)$ over the attributes that are present in the partial profile $\Pi$ equals 1.

Now, assume that the weighted attribute-level maxdiff model, (19), holds for the best-worst attribute-level choice probabilities on the full profiles $P$ and that the best-worst attribute-level choice probabilities for each partial profile $\Pi$ are represented by the attribute-level maxdiff model with the same scale $\widetilde{b}$ but with weights (exponents) $\beta_{i}(\Pi)$ given by $(25)$.

Then, by assumption, the relative importance weights in (25) satisfy the constant ratio property of the Luce (or MNL) choice model (Luce, 1959/2005) - 
in particular, for every partial profile $\Pi$ and all attributes $i, j \in\{1, \ldots, m\}$ that occur in the partial profile $\Pi$,

$$
\frac{\beta_{i}(\Pi)}{\beta_{j}(\Pi)}=\frac{\beta_{i}}{\beta_{j}},
$$

where $\beta_{k}, k=1, \ldots, m$, are the weights when all the attributes are present. Thus, one can fit the best-worst attribute data for the various (partial) profiles assuming that the importance weights are constrained by (26) and that the utility values remain constant across (partial) profiles.

Norman (1980) developed essentially this approach, but in terms of variance accounted for by each attribute for each partial profile context (in a rating task), rather than (best-worst attribute-level) choice. He found support for the constant ratio rule in his own study of the rating of job candidates, plus 22 other data sets.

\section{Discussion and Conclusions}

The results in this paper extend Marley and Louviere's (2005) results for best and best-worst choice among objects to best and best-worst choices among levels of attributes in experimentally designed profiles. The archetypal application of our work would be to one-at-a-time conjoint measurement/conjoint analysis profiles where individuals are asked to evaluate several combinations of attribute levels constructed from an orthogonal array (e.g., Green 1974; Louviere 1988; Luce \& Tukey 1969). Our results demonstrate that if individuals make choices in best-worst choice tasks according to the models derived in this paper, one can measure the attribute levels of all attributes on a common underlying scale, allowing inter-dimensional latent scale comparisons within individuals. We believe that our results constitute the first demonstration that an elicitation task 
and associated choice model can be used to estimate subjective attribute level values on such a common scale.

We hope that eventually the theory and data of the type described in this paper can be used to separate attribute weights and utility values in various probabilistic models of best and best-worst attribute-level choices. The challenge is to demonstrate that there are models and designs like the ones described in the paper that, in theory and in practice, are sufficient to separate these factors. Norman's (1980) early contribution, and Louviere and Islam's (2007) recent work, encourage us in our belief that this is possible [ajm: delete: , even whilst we acknowledge the long history of failed attempts in this area]. Marley (1991) summarizes earlier work on context dependent probabilistic choice models that may suggest ways to approach the theoretical challenge. The associated empirical challenge will be to collect sufficiently reliable data to evaluate the models.

[ajm: delete the following paragraph, as per Referee 1 (or revise and place elsewhere):] Finally, it is worth noting that the best-worst choice tasks discussed in the paper produce sufficient data to allow one to derive estimates for individuals. Naturally, the "quality" of the resulting estimates will depend on the quantity (the sample size) and quality (reliability and validity) of the choice data. However, the empirical experience of one of the authors is that typically the resulting estimates are sufficient to allow a wide variety of additional analyses to be performed on the estimates, such as various clustering methods to identify groups, testing for relationships with covariates that describe differences in individuals and the like. Thus, it is likely that the attribute-level estimates described in this paper will eventually be as useful as the object based estimates described by Marley and Louviere (2005) that now are routinely used in many marketing and survey research applications.] 


\section{Proofs}

\section{Theorem 3}

Proof. It is routine to check that if set of best choice probabilities on a finite set $T$ satisfies a set-dependent Luce model, (1), then it satisfies the set-dependent constant ratio rule, (2), and that if it satisfies Luce's model, then it satisfies the constant ratio rule. So it remains to prove the converses.

First assume that the set-dependent constant ratio rule holds and for $z \in T$, let

$$
b_{T}(z)=B_{T}(z)
$$

and for $\{r, s\} \subseteq Z \subseteq T,|Z| \geq 2$, let

$$
\varphi_{T}(Z)=\frac{\log \frac{B_{Z}(r)}{B_{Z}(s)}}{\log \frac{B_{T}(r)}{B_{T}(s)}} \quad(r \neq s) .
$$

The function $\varphi_{T}$ is well-defined since, by the set-dependent constant ratio rule, (2), it is independent of the selected elements $r, s \in Z, r \neq s$. Note that $\varphi_{T}(T)=1$. Also, $T$ is fixed throughout the development so from here on we write $b(z)$ for $b_{T}(z)$ and $\varphi(Z)$ for $\varphi_{T}(Z)$. Using all of these properties, we have for $X \subseteq T,|X| \geq 2, x, y \in X, x \neq y$,

$$
\begin{aligned}
\varphi(X) \log \frac{b(y)}{b(x)} & =\frac{\log \frac{B_{X}(y)}{B_{X}(x)}}{\log \frac{B_{T}(y)}{B_{T}(x)}} \cdot \log \frac{B_{T}(y)}{B_{T}(x)} \\
& =\log \frac{B_{X}(y)}{B_{X}(x)}
\end{aligned}
$$

i.e. for $x \neq y$,

$$
\frac{B_{X}(y)}{B_{X}(x)}=\left[\frac{b(y)}{b(x)}\right]^{\varphi(X)}
$$

Clearly, the above formula also holds when $x=y$, and summing over $y \in X$, we 
have

$$
\begin{aligned}
\frac{1}{B_{X}(x)} & =\sum_{y \in X} \frac{B_{X}(y)}{B_{X}(x)}=\sum_{y \in X}\left[\frac{b(y)}{b(x)}\right]^{\varphi(X)} \\
& =\frac{\sum_{y \in X} b(y)^{\varphi(X)}}{b(x)^{\varphi(X)}}
\end{aligned}
$$

i.e.,

$$
B_{X}(x)=\frac{b(x)^{\varphi(X)}}{\sum_{y \in X} b(y)^{\varphi(X)}},
$$

which is the representation for a set-dependent Luce model.

Now, we show that $b$ is a loginterval scale and $\varphi$ a ratio scale with the unit of $\varphi$ the reciprocal of the unit of $b$. So suppose that $b^{\prime}, \varphi^{\prime}$ are another pair of functions such that for each $x \in X \subseteq T$,

$$
B_{X}(x)=\frac{b^{\prime}(x)^{\varphi^{\prime}(X)}}{\sum_{y \in X} b^{\prime}(y)^{\varphi^{\prime}(X)}} .
$$

In particular, for each $x \in T$,

$$
\begin{aligned}
b(x) & =B_{T}(x) \\
& =\frac{b^{\prime}(x)^{\varphi^{\prime}(T)}}{\sum_{y \in T} b^{\prime}(y)^{\varphi^{\prime}(T)}} .
\end{aligned}
$$

Letting

$$
a^{\prime}=\frac{1}{\sum_{y \in T} b^{\prime}(y)^{\varphi^{\prime}(T)}}, \alpha^{\prime}=\varphi^{\prime}(T)
$$

then we have

$$
b=a^{\prime}\left(b^{\prime}\right)^{\alpha^{\prime}},
$$

i.e., $b$ is a loginterval scale. 
Also, using (29) with (27), (28), we obtain: for $x, y \in X, x \neq y$,

$$
\left(\frac{b^{\prime}(x)}{b^{\prime}(y)}\right)^{\varphi^{\prime}(T) \varphi(X)}=\left(\frac{b(x)}{b(y)}\right)^{\varphi(X)}=\frac{B_{X}(x)}{B_{X}(y)}=\left(\frac{b^{\prime}(x)}{b^{\prime}(y)}\right)^{\varphi^{\prime}(X)}
$$

i.e., $\varphi=\frac{\varphi^{\prime}}{\varphi^{\prime}(T)}=\frac{\varphi^{\prime}}{\alpha^{\prime}}$ is a ratio scale, with the unit of $\varphi$, i.e., $1 / \alpha^{\prime}$, the reciprocal of the unit of $b$, i.e., $\alpha^{\prime}$.

Finally, when the constant ratio rule holds, the above proof gives, for all $Z \subseteq T, \varphi(Z)=\varphi_{T}(Z)=1$, and the representation reduces to that of Luce's model.

\section{Theorem 8}

Proof. Remember that we are assuming that 3 -reversibility hold vacuously when $|T| \geq 2$.

It is routine to check that if set of best-worst choice probabilities on a finite set $T,|T| \geq 2$, satisfies a set-dependent maxdiff model, (3), then it satisfies the set-dependent best-worst constant ratio rule, 2-invertibility and 3-reversibility, and that if it satisfies the maxdiff model, then it satisfies the best-worst constant ratio rule, 2 -invertibility and 3 -reversibility. So it remains to prove the converses.

Fix $x_{o} \in T$ and for arbitrary $z \in T$, let

$$
b_{T}(z)=\left\{\begin{array}{clc}
1 & \text { if } \quad z=x_{o} . \\
\left(\frac{B W_{T}\left(z, x_{0}\right)}{B W_{T}\left(x_{0}, z\right)}\right)^{\frac{1}{2}} & \text { if } \quad z \neq x_{0} .
\end{array}\right.
$$

and for $Z \subseteq T,|Z| \geq 2, r, s \in Z, r \neq s$, let

$$
\phi_{T}(Z)=\frac{\log \frac{B_{Z}(r, s)}{B_{Z}(s, r)}}{\log \frac{B_{T}(r, s)}{B_{T}(s, r)}} \quad(r \neq s)
$$

The function $\phi_{T}$ is well-defined since, by (4), it is independent of the selected 
elements $r, s \in Z, r \neq s$. Note that $\phi_{T}(T)=1$. Also, $T$ is fixed throughout the development so from here on we write $b(z)$ for $b_{T}(z)$ and $\phi(Z)$ for $\phi_{T}(Z)$.

We show first that (30) with 3-reversibility, (6), implies that for all $r, s \in T$, $r \neq s$

$$
\frac{b(r)}{b(s)}=\left(\frac{B W_{T}(r, s)}{B W_{T}(s, r)}\right)^{\frac{1}{2}}
$$

For clarity, we separate the case $|T|=2$ from $|T| \geq 3$.

Case 1. $|T|=2$.

First, using (30), it is easily checked that (32) holds if $r$ or $s$ is $x_{0}$ and so (32) holds if $|T|=2$.

Case 2. $|T| \geq 3$.

Again, using (30), it is easily checked that (32) holds if $r$ or $s$ is $x_{0}$. So consider $r \neq x_{0}, s \neq x_{0}$. Then] using (30) with 3 -reversibility, (6), for $X=T$, we have

$$
\begin{aligned}
\frac{b(r)}{b(s)} & =\left(\frac{B W_{T}\left(r, x_{0}\right)}{B W_{T}\left(x_{0}, r\right)} \cdot \frac{B W_{T}\left(x_{0}, s\right)}{B W_{T}\left(s, x_{0}\right)}\right)^{\frac{1}{2}} \\
& =\left(\frac{B W_{T}(r, s)}{B W_{T}(s, r)}\right)^{\frac{1}{2}}
\end{aligned}
$$

and so (32) holds for all $r, s \in T$.

We now use the properties of $b,(32)$, and the definition of $\phi,(31)$, to obtain the representation for the set-dependent maxdiff model, (3), for arbitrary $T,|T|$ $\geq 2$.

We have: for $X \subseteq T,|X| \geq 2, r, s \in X$,

$$
\begin{aligned}
\phi(X) \log \frac{b(r)}{b(s)} & =\frac{\log \frac{B W_{X}(r, s)}{B W_{X}(s, r)}}{\log \frac{B W_{T}(r, s)}{B W_{T}(s, r)}} \cdot \frac{1}{2} \log \left(\frac{B W_{T}(r, s)}{B W_{T}(s, r)}\right) \\
& =\frac{1}{2} \cdot \log \frac{B W_{X}(r, s)}{B W_{X}(s, r)} .
\end{aligned}
$$


i.e.,

$$
\left(\frac{b(r)}{b(s)}\right)^{\phi(X)}=\left(\frac{B W_{X}(r, s)}{B W_{X}(s, r)}\right)^{\frac{1}{2}} .
$$

Then, for given $x, y \in X, x \neq y$, we have

$$
\begin{aligned}
\frac{\sum_{r, s \in X}^{r \neq s}\left(\frac{b(r)}{b(s)}\right)^{\phi(X)}}{\left(\frac{b(x)}{b(y)}\right)^{\phi(X)}} & =\frac{\sum_{r, s \in X}\left(\frac{B W_{X}(r, s)}{B \neq \neq}\left(\frac{1}{B W_{X}(s, r)}\right)^{\frac{1}{2}}\right.}{\left(\frac{B W_{X}(x, y)}{B W_{X}(y, x)}\right)^{\frac{1}{2}}} \\
& =\frac{1}{B W_{X}(x, y)} \cdot \sum_{\substack{r, s \in X \\
r \neq s}}\left[B W_{X}(x, y) B W_{X}(y, x)\right]^{\frac{1}{2}}\left(\frac{B W_{X}(r, s)}{B W_{X}(s, r)}\right)^{\frac{1}{2}} .
\end{aligned}
$$

Thus, if we can show that

$$
\sum_{\substack{r, s \in X \\ r \neq s}}\left[B W_{X}(x, y) B W_{X}(y, x)\right]^{\frac{1}{2}}\left(\frac{B W_{X}(r, s)}{B W_{X}(s, r)}\right)^{\frac{1}{2}}=1,
$$

then (33) gives, for $x \neq y$,

$$
B W_{X}(x, y)=\frac{\left(\frac{b(x)}{b(y)}\right)^{\phi(X)}}{\sum_{\substack{r, s \in X \\ r \neq s}}\left(\frac{b(r)}{b(s)}\right)^{\phi(X)}}
$$

which is the representation for the set-dependent maxdiff model, (3). So we now show that (34) holds. Using 2-invertibility, (5),

$$
\begin{aligned}
& \sum_{\substack{r, s \in X \\
r \neq s}}\left[B W_{X}(x, y) B W_{X}(y, x)\right]^{\frac{1}{2}}\left(\frac{B W_{X}(r, s)}{B W_{X}(s, r)}\right)^{\frac{1}{2}} \\
& =\sum_{\substack{r, s \in X \\
r \neq s}}\left[B W_{X}(r, s) B W_{X}(s, r)\right]^{\frac{1}{2}}\left(\frac{B W_{X}(r, s)}{B W_{X}(s, r)}\right)^{\frac{1}{2}} \\
& =\sum_{\substack{r, s \in X \\
r \neq s}} B W_{X}(r, s)=1,
\end{aligned}
$$


as desired.

We now show that $b$ is a loginterval scale and $\phi$ a ratio scale. So suppose that $b^{\prime}, \phi^{\prime}$ are another pair of such functions. We first consider $z \in T-\left\{x_{0}\right\}$, and consider the special case $z=x_{0}$ at the end of the argument. For $z \neq x_{0}$,

$$
\begin{aligned}
b(z) & =\left(\frac{B W_{T}\left(z, x_{0}\right)}{B W_{T}\left(x_{0}, z\right)}\right)^{\frac{1}{2}} \\
& =\left[\frac{\left(\frac{b^{\prime}(z)}{b^{\prime}\left(x_{0}\right)}\right)^{\phi^{\prime}(T)}}{\left(\frac{b^{\prime}\left(x_{0}\right)}{b^{\prime}(z)}\right)^{\phi^{\prime}(T)}}\right]^{\frac{1}{2}} \\
& =\left[\left(\frac{b^{\prime}(z)}{b^{\prime}\left(x_{0}\right)}\right)^{2 \phi^{\prime}(T)}\right]^{\frac{1}{2}} \\
& =\frac{b^{\prime}(z)^{\phi^{\prime}(T)}}{b^{\prime}\left(x_{0}\right)^{\phi^{\prime}(T)}} .
\end{aligned}
$$

Now let

$$
a^{\prime}=\frac{1}{b^{\prime}\left(x_{0}\right)^{\phi^{\prime}(T)}}, \alpha^{\prime}=\phi^{\prime}(T)
$$

Then, remembering that we have assumed that $z \neq x_{0}$, we have $b(z)=a^{\prime}\left[b^{\prime}(z)\right]^{\alpha^{\prime}}$ for $z \neq x_{0}$ and $\phi=\alpha \phi^{\prime}$. However, it is obvious, using the fact that $b\left(x_{0}\right)=1$, that we also have $b\left(x_{0}\right)=a^{\prime}\left[b^{\prime}\left(x_{0}\right)\right]^{\alpha^{\prime}}$. Therefore

$$
b=a^{\prime}\left(b^{\prime}\right)^{\alpha^{\prime}}
$$

i.e., $b$ is a loginterval scale.

Also, using (35) with the set-dependent maxdiff model, (3), for each of the pairs $(b, \phi)$ and $\left(b^{\prime}, \phi^{\prime}\right)$, we obtain: for $x, y \in X, x \neq y$,

$$
\left(\frac{b^{\prime}(x)}{b^{\prime}(y)}\right)^{2 \phi^{\prime}(X)}=\frac{B W_{X}(x, y)}{B W_{X}(y, x)}=\left(\frac{b(x)}{b(y)}\right)^{2 \phi(X)}=\left(\frac{b^{\prime}(x)}{b^{\prime}(y)}\right)^{2 \phi^{\prime}(T) \phi(X)}
$$

i.e., $\phi=\frac{\phi^{\prime}}{\phi^{\prime}(T)}=\frac{\phi^{\prime}}{\alpha^{\prime}}$ is a ratio scale, with the unit of $\phi$, i.e., $1 / \alpha^{\prime}$, the reciprocal 
of the unit of $b$, i.e., $\alpha^{\prime}$.

Finally, when the best-worst constant ratio rule holds, the above proof gives, for all $Z \subseteq T, \phi(Z)=\phi_{T}(Z)=1$, so the representation reduces to the maxdiff model. Also, using notation as in the above proof, this implies that $\phi(T)=1=$ $\phi^{\prime}(T)$ for any pair of representations. However, in that proof $\phi^{\prime}(T)=\alpha^{\prime}$, hence $\alpha^{\prime}=1$, so (35) reduces to $b=a^{\prime} b^{\prime}$, i.e., $b$ is a ratio scale.

\section{Theorem 11}

Proof. It is routine to check that if a set of best choice probabilities on a finite set of profiles $P$ satisfies an attribute-level Luce model, (8), with $b$ a ratio scale, then it satisfies $2-, 3-$, and $4-$ reversibility, Def. 10. So it remains to prove the converse.

Let $*=\left(*_{1}, \ldots, *_{m}\right)$ denote a fixed vector of attribute-levels and so $*_{j}$ is the level of $*$ on the $j$ th attribute. For any attribute-level $v_{g}$, let $* \backslash v_{g}$ denote the vector that agrees with $*$ except - possibly - on attribute-level $v_{g}$. The reason for the parenthetic "possibly" is that if $*_{g} \neq v_{g}$ then $* \backslash v_{g}$ differs from $*$, whereas if $*_{g}=v_{g}$ then $* \backslash v_{g}$ is identical to $*$ - and each case occurs.

Now define the scale $b$ by the following ${ }^{9}$ : for $g \in M$

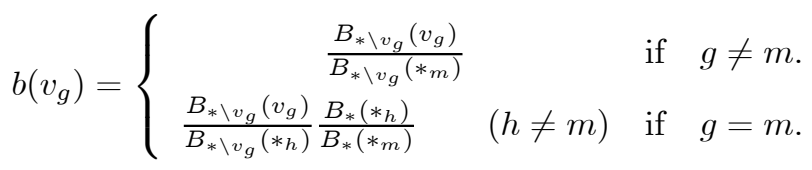

Note that when $g=m$ and $v_{g}=*_{m}$, the second line of $(36)$ gives $b\left(v_{g}\right)=$ $b\left(*_{m}\right)=1$.

1. We first show that 2-reversibility, (9), implies that (36) does not depend

\footnotetext{
${ }^{9}$ We could define the scale values by the single condition

$$
b\left(v_{g}\right)=\frac{B_{* \backslash v_{g}}\left(v_{g}\right)}{B_{* \backslash v_{g}}\left(*_{h}\right)} \frac{B_{*}\left(*_{h}\right)}{B_{*}\left(*_{m}\right)} \quad(h \neq g),
$$

which includes our split condition as a special case. However, the proof would then need to be carried out using " 5 -reversibility", the obvious generalization of $n$-reversibility, $n=2,3,4$.
} 
on the selected attribute $h \neq m$ for the case $g=m$. (There is nothing to prove when $g \neq m$.)

If $m=2$, then the only possible value for $h$ is $h=1$ and so there is nothing to prove. For $m>2$, take $h \neq h^{\prime}$ with each different from $m$. Then, remembering that $g=m$, the value of $b\left(v_{g}\right)$ is independent of $h$ iff, when $h \neq h^{\prime}$,

$$
\frac{B_{* \backslash v_{m}}\left(v_{m}\right)}{B_{* \backslash v_{m}}\left(*_{h}\right)} \frac{B_{*}\left(*_{h}\right)}{B_{*}\left(*_{m}\right)}=\frac{B_{* \backslash v_{m}}\left(v_{m}\right)}{B_{* \backslash v_{m}}\left(*_{h^{\prime}}\right)} \frac{B_{*}\left(*_{h^{\prime}}\right)}{B_{*}\left(*_{m}\right)},
$$

which reduces to

$$
B_{*}\left(*_{h}\right) B_{* \backslash v_{m}}\left(*_{h^{\prime}}\right)=B_{*}\left(*_{h^{\prime}}\right) B_{* \backslash v_{m}}\left(*_{h}\right) .
$$

However, this equality holds as it is the special case of 2 -reversibility, (9), with $\mathbf{r}=*, \mathbf{s}=* \backslash v_{m}, i=h, j=h^{\prime}$, and so $r_{i}=s_{i}=*_{h}$ and $r_{j}=s_{j}=*_{h^{\prime}}$.

2. Next, we show that (36) with 3- and 4-reversibility, (10) and (11), implies that for all $\mathbf{v}$,

$$
\frac{b\left(v_{i}\right)}{b\left(v_{j}\right)}=\frac{B_{\mathbf{v}}\left(v_{i}\right)}{B_{\mathbf{v}}\left(v_{j}\right)}
$$

and from this obtain the desired representation.

It is clear that (37) holds when $i=j$, so in the remainder of the proof we assume that $i \neq j$.

Case 1. $i=m, j \neq m$. (The case $i \neq m, j=m$ is equivalent to this one.) This case includes that where $m=2$, since in that case $i \neq j$ forces $i=m, j \neq m$ (or the equivalent $i \neq m, j=m$ ). Now, consider 4 -reversibility, (11), with $\mathbf{r}=* \backslash v_{i}, \mathbf{s}=*, \mathbf{t}=* \backslash v_{j}, \mathbf{u}=\mathbf{v}$, and let $k=m, l=j$. Then, because $i=m, j \neq m$, we have, as required by 4 -reversibility, $i \neq j, j \neq k$ and $k \neq l$. Also, $r_{i}=u_{i}=v_{i}, s_{j}=r_{j}=*_{j}, t_{k}=s_{k}=*_{m}, u_{l}=t_{l}=v_{j}$ and so 
4 -reversibility becomes

$$
\begin{aligned}
& B_{* \backslash v_{i}}\left(v_{i}\right) B_{*}\left(*_{j}\right) B_{* \backslash v_{j}}\left(*_{m}\right) B_{\mathbf{v}}\left(v_{j}\right) \\
& =B_{* \backslash v_{i}}\left(*_{j}\right) B_{*}\left(*_{m}\right) B_{* \backslash v_{j}}\left(v_{j}\right) B_{\mathbf{v}}\left(v_{i}\right)
\end{aligned}
$$

The above equality, with (36) for $g=i=m, h=j \neq m$ and, separately, for $g=j \neq m$, gives

$$
\frac{b\left(v_{i}\right)}{b\left(v_{j}\right)}=\left(\frac{B_{* \backslash v_{i}}\left(v_{i}\right)}{B_{* \backslash v_{i}}\left(*_{j}\right)} \frac{B_{*}\left(*_{j}\right)}{B_{*}\left(*_{m}\right)}\right) \frac{B_{* \backslash v_{j}}\left(*_{m}\right)}{B_{* \backslash v_{j}}\left(v_{j}\right)}=\frac{B_{\mathbf{v}}\left(v_{i}\right)}{B_{\mathbf{v}}\left(v_{j}\right)}
$$

Case 2. $i \neq m, j \neq m$. We showed that if $m=2$, then Case 1 holds, so here we can assume that $m \geq 3$. Now, 3-reversibility, (10), with $k=m, \mathbf{r}=\mathbf{v}$, $\mathbf{s}=* \backslash v_{j}, \mathbf{t}=* \backslash v_{i}$ and so $r_{i}=v_{i}, s_{j}=v_{j}, t_{k}=s_{k}=*_{m}$, gives $i \neq j, j \neq k$ and

$$
\begin{aligned}
& B_{\mathbf{v}}\left(v_{i}\right) B_{* \backslash v_{j}}\left(v_{j}\right) B_{* \backslash v_{i}}\left(*_{m}\right) \\
& \quad=B_{\mathbf{v}}\left(v_{j}\right) B_{* \backslash v_{j}}\left(*_{m}\right) B_{* \backslash v_{i}}\left(v_{i}\right),
\end{aligned}
$$

which with (36) for $g=i \neq m$, and, separately, for $g=j \neq m$, gives

$$
\frac{b\left(v_{i}\right)}{b\left(v_{j}\right)}=\frac{B_{* \backslash v_{i}}\left(v_{i}\right) B_{* \backslash v_{j}}\left(*_{m}\right)}{B_{* \backslash v_{i}}\left(*_{m}\right) B_{* \backslash v_{j}}\left(v_{j}\right)}=\frac{B_{\mathbf{v}}\left(v_{i}\right)}{B_{\mathbf{v}}\left(v_{j}\right)} .
$$

3. Using the result that (37) holds for all $i, j$, we have for each profile $\mathbf{z}=\left(\mathbf{z}_{1}, \ldots, z_{m}\right)$,

$$
\frac{\sum_{j \in M} b\left(z_{j}\right)}{b\left(z_{i}\right)}=\sum_{j \in M} \frac{b\left(z_{j}\right)}{b\left(z_{i}\right)}=\sum_{j \in M} \frac{B_{\mathbf{z}}\left(z_{j}\right)}{B_{\mathbf{z}}\left(z_{i}\right)}=\frac{\sum_{j \in M} B_{\mathbf{z}}\left(z_{j}\right)}{B_{\mathbf{z}}\left(z_{i}\right)}=\frac{1}{B_{\mathbf{z}}\left(z_{i}\right)}
$$


i.e.,

$$
B_{\mathbf{z}}\left(z_{i}\right)=\frac{b\left(z_{i}\right)}{\sum_{j \in M} b\left(z_{j}\right)},
$$

which is the representation of the attribute-level Luce model, (8).

4. Finally, we show that $b$ is a ratio scale. If $b^{\prime}$ is another such function, then when $j \neq m$,

$$
\begin{aligned}
b\left(v_{j}\right) & =\frac{B_{* \backslash v_{j}}\left(v_{j}\right)}{B_{* \backslash v_{j}}\left(*_{m}\right)} \\
& =\frac{b^{\prime}\left(v_{j}\right)}{b^{\prime}\left(*_{m}\right)} \\
& =a^{\prime} b^{\prime}\left(v_{j}\right),
\end{aligned}
$$

with $a^{\prime}=1 / b^{\prime}\left(*_{m}\right)$.

Similarly, when $j=m$, there is $h \neq m$ with

$$
\begin{aligned}
b\left(v_{j}\right) & =\frac{B_{* \backslash v_{h}}\left(v_{j}\right)}{B_{* \backslash v_{h}}\left(*_{h}\right)} \frac{B_{*}\left(*_{h}\right)}{B_{*}\left(*_{m}\right)} \\
& =\frac{b^{\prime}\left(v_{j}\right)}{b^{\prime}\left(*_{h}\right)} \frac{b^{\prime}\left(*_{h}\right)}{b^{\prime}\left(*_{m}\right)} \\
& =\frac{b^{\prime}\left(v_{j}\right)}{b^{\prime}\left(*_{m}\right)} \\
& =a^{\prime} b^{\prime}\left(v_{j}\right),
\end{aligned}
$$

with $a^{\prime}=1 / b^{\prime}\left(*_{m}\right)$.

Thus, $b=a^{\prime} b^{\prime}$, so $b$ is a ratio scale.

\section{Theorem 15}

Proof. It is routine to check that if a set of best-worst choice probabilities on a finite set of profiles $P$ satisfies a maxdiff weighted attribute-level choice model, (12), then it satisfies 2-invertibility, Def. 13, and 3- and 4-reversibility, Def. 14. So it remains to prove the converse.

As in the proof of Theorem 11, let $*$ denote a fixed vector of attribute-levels, 
and define the scale $b$ by the following:

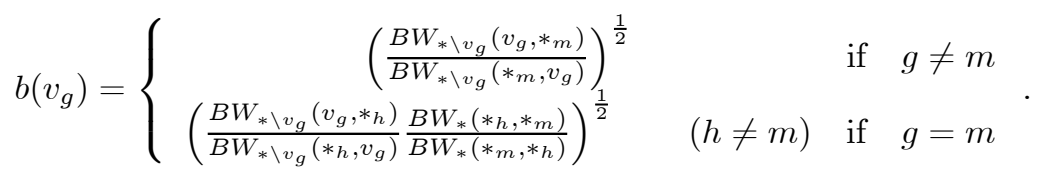

Note that, when $g=m$ and $v_{g}=*_{m}$, the second line of (38) gives $b\left(v_{g}\right)=$ $b\left(*_{m}\right)=1$.

1. We show first that a special case of 4-reversibility, (15), implies that (38) does not depend on the selected attribute $h \neq m$ for the case $g=m$. (There is nothing to prove when $g \neq m$ ).

If $m=2$, then the only possible value for $h$ is $h=1$ and so there is nothing to prove. For $m>2$, take $h \neq h^{\prime}$ with each different from $m$. Then the value of $b\left(v_{g}\right)$ is independent of $h$ iff, when $h \neq h^{\prime}$ and $g=m$,

$$
\frac{B W_{* \backslash v_{m}}\left(v_{m}, *_{h}\right)}{B W_{* \backslash v_{m}}\left(*_{h}, v_{m}\right)} \frac{B W_{*}\left(*_{h}, *_{m}\right)}{B W_{*}\left(*_{m}, *_{h}\right)}=\frac{B W_{* \backslash v_{m}}\left(v_{m}, *_{h^{\prime}}\right)}{B W_{* \backslash v_{m}}\left(*_{h^{\prime}}, v_{m}\right)} \frac{B W_{*}\left(*_{h^{\prime}}, *_{m}\right)}{B W_{*}\left(*_{m}, *_{h^{\prime}}\right)},
$$

i.e., iff

$$
\begin{aligned}
& B W_{* \backslash v_{m}}\left(v_{m}, *_{h}\right) B W_{*}\left(*_{h}, *_{m}\right) B W_{*}\left(*_{m}, *_{h^{\prime}}\right) B W_{* \backslash v_{m}}\left(*_{h^{\prime}}, v_{m}\right) \\
& \quad=B W_{* \backslash v_{m}}\left(v_{m}, *_{h^{\prime}}\right) B W_{*}\left(*_{h^{\prime}}, *_{m}\right) B W_{*}\left(*_{m}, *_{h}\right) B W_{* \backslash v_{m}}\left(*_{h}, v_{m}\right)
\end{aligned}
$$

However, the equality holds as it corresponds to the special case of 4-reversibility, (15), with $\mathbf{u}=\mathbf{r}=* \backslash v_{m}, \mathbf{s}=\mathbf{t}=*$ and $j=h \neq m, l=h^{\prime} \neq m, i=k=m$. In particular, we have $i \neq j, j \neq k, k \neq l$, as required in the definition of 4-reversibility.

2. Now we show that (38) with 3- and 4-reversibility, (14) and (15), implies that, for all $\mathbf{v}$ and $v_{i}, v_{j}$, 


$$
\frac{b\left(v_{i}\right)}{b\left(v_{j}\right)}=\left(\frac{B W_{\mathbf{v}}\left(v_{i}, v_{j}\right)}{B W_{\mathbf{v}}\left(v_{j}, v_{i}\right)}\right)^{\frac{1}{2}}
$$

It is clear that (39) holds when $i=j$, so in the remainder of the proof we assume that $i \neq j$.

Case 1. $i=m, j \neq m$. (The remaining case, $i \neq m, j=m$, is equivalent to this case). This case includes that where $m=2$ since in that case $i \neq j$ implies $i=m, j \neq m$ (or the equivalent $i \neq m, j=m$ ). Now, consider 4-reversibility, (15), with $\mathbf{r}=\mathbf{v}, \mathbf{s}=* \backslash v_{j}, \mathbf{t}=*, \mathbf{u}=* \backslash v_{i}$ and $k=m, l \neq m$. Then $r_{i}=u_{i}=v_{i}, s_{j}=r_{j}=v_{j}, t_{k}=s_{k}=*_{m}, u_{l}=t_{l}=*_{l}, i \neq j, j \neq k, k \neq l$, which gives

$$
\begin{aligned}
& B W_{\mathbf{v}}\left(v_{i}, v_{j}\right) B W_{* \backslash v_{j}}\left(v_{j}, *_{m}\right) B W_{*}\left(*_{m}, *_{l}\right) B W_{* \backslash v_{i}}\left(*_{l}, v_{i}\right) \\
& \quad=B W_{* \backslash v_{i}}\left(v_{i}, *_{l}\right) B W_{*}\left(*_{l}, *_{m}\right) B W_{* \backslash v_{j}}\left(*_{m}, v_{j}\right) B W_{\mathbf{v}}\left(v_{j}, v_{i}\right) .
\end{aligned}
$$

The above equality, and (38) with $g=i=m, h=l \neq m$ and, separately, $g=j \neq m$, together give

$$
\begin{aligned}
\frac{b\left(v_{i}\right)}{b\left(v_{j}\right)} & =\left(\frac{B W_{* \backslash v_{i}}\left(v_{i}, *_{l}\right)}{B W_{* \backslash v_{i}}\left(*_{l}, v_{i}\right)} \frac{B W_{*}\left(*_{l}, *_{m}\right)}{B W_{*}\left(*_{m}, *_{l}\right)}\right)^{\frac{1}{2}}\left(\frac{B W_{* \backslash v_{j}}\left(*_{m}, v_{j}\right)}{B W_{* \backslash v_{j}}\left(v_{j}, *_{m}\right)}\right)^{\frac{1}{2}} \\
& =\left(\frac{B W_{\mathbf{v}}\left(v_{i}, v_{j}\right)}{B W_{\mathbf{v}}\left(v_{j}, v_{i}\right)}\right)^{\frac{1}{2}}
\end{aligned}
$$

Case 2. $i \neq m, j \neq m$. We showed that if $m=2$, then Case 1 holds, so here we can assume that $m \geq 3$. Now, consider 3 -reversibility, (14), with $\mathbf{r}=\mathbf{v}$, $\mathbf{s}=* \backslash v_{j}, \mathbf{t}=* \backslash v_{i}$ and $k=m$. Then $r_{i}=t_{i}=v_{i}, s_{j}=r_{j}=v_{j}, s_{k}=t_{k}=*_{m}$, 
$i \neq j, j \neq k$, which gives

$$
\begin{aligned}
& B W_{\mathbf{v}}\left(v_{i}, v_{j}\right) B W_{* \backslash v_{j}}\left(v_{j}, *_{m}\right) B W_{* \backslash v_{i}}\left(*_{m}, v_{i}\right) \\
& \quad=B W_{* \backslash v_{i}}\left(v_{i}, *_{m}\right) B W_{* \backslash v_{j}}\left(*_{m}, v_{j}\right) B W_{\mathbf{v}}\left(v_{j}, v_{i}\right) .
\end{aligned}
$$

The above equality, with (38) and $g=i \neq m$ and, separately, $g=j \neq m$, gives

$$
\begin{aligned}
\frac{b\left(v_{i}\right)}{b\left(v_{j}\right)} & =\left(\frac{B W_{* \backslash v_{i}}\left(v_{i}, *_{m}\right)}{B W_{* \backslash v_{i}}\left(*_{m}, v_{i}\right)}\right)^{\frac{1}{2}}\left(\frac{B W_{* \backslash v_{j}}\left(*_{m}, v_{j}\right)}{B W_{* \backslash v_{j}}\left(v_{j}, *_{m}\right)}\right)^{\frac{1}{2}} \\
& =\left(\frac{B W_{\mathbf{v}}\left(v_{i}, v_{j}\right)}{B W_{\mathbf{v}}\left(v_{j}, v_{i}\right)}\right)^{\frac{1}{2}} .
\end{aligned}
$$

Thus, we have (39) for all $v$ and $i, j \in M, i \neq j$.

3. Using the fact that (39) holds, we have for a each profile $\mathbf{z}=\left(\mathbf{z}_{1}, \ldots, z_{m}\right)$,

$$
\begin{aligned}
\left.\frac{\sum_{\substack{k, l \in M \\
k \neq l}}^{\left(\frac{b\left(v_{i}\right)}{b\left(v_{j}\right)}\right)}=}{\left(v_{k}\right)}\right) & \frac{\sum_{\substack{k, l \in M \\
k \neq l}}\left(\frac{B W_{\mathbf{v}}\left(v_{k}, v_{l}\right)}{B W_{\mathbf{v}}\left(v_{l}, v_{k}\right)}\right)^{\frac{1}{2}}}{\left(\frac{B W_{\mathbf{v}}\left(v_{i}, v_{j}\right)}{B W_{\mathbf{v}}\left(v_{j}, v_{i}\right)}\right)^{\frac{1}{2}}} \\
= & \frac{1}{B W_{\mathbf{v}}\left(v_{i}, v_{j}\right)} \\
& \times \sum_{\substack{k, l \in M \\
k \neq l}}\left[B W_{\mathbf{v}}\left(v_{i}, v_{j}\right) B W_{\mathbf{v}}\left(v_{j}, v_{i}\right)\right]^{\frac{1}{2}}\left(\frac{B W_{\mathbf{v}}\left(v_{k}, v_{l}\right)}{B W_{\mathbf{v}}\left(v_{l}, v_{k}\right)}\right)^{\frac{1}{2}} .
\end{aligned}
$$

Thus, if we can show that

$$
\sum_{\substack{k, l \in M \\ k \neq l}}\left[B W_{\mathbf{v}}\left(v_{i}, v_{j}\right) B W_{\mathbf{v}}\left(v_{j}, v_{i}\right)\right]^{\frac{1}{2}}\left(\frac{B W_{\mathbf{v}}\left(v_{k}, v_{l}\right)}{B W_{\mathbf{v}}\left(v_{l}, v_{k}\right)}\right)^{\frac{1}{2}}=1,
$$


then (40) gives

$$
B W_{\mathbf{v}}\left(v_{i}, v_{j}\right)=\frac{\left(\frac{b\left(v_{i}\right)}{b\left(v_{j}\right)}\right)}{\sum_{\substack{k, l \in M \\ k \neq l}}\left(\frac{b\left(v_{k}\right)}{b\left(v_{l}\right)}\right)}
$$

which is the representation of the attribute-level maxdiff model, (12). So we now show that (41) holds. Using 2-invertibility, (13),

$$
\begin{aligned}
& \sum_{\substack{k, l \in M \\
k \neq l}}\left[B W_{\mathbf{v}}\left(v_{i}, v_{j}\right) B W_{\mathbf{v}}\left(v_{j}, v_{i}\right)\right]^{\frac{1}{2}}\left(\frac{B W_{\mathbf{v}}\left(v_{k}, v_{l}\right)}{B W_{\mathbf{v}}\left(v_{l}, v_{k}\right)}\right)^{\frac{1}{2}} \\
& =\sum_{\substack{k, l \in M \\
k \neq l}}\left[B W_{\mathbf{v}}\left(v_{k}, v_{l}\right) B W_{\mathbf{v}}\left(v_{l}, v_{k}\right)\right]^{\frac{1}{2}}\left(\frac{B W_{\mathbf{v}}\left(v_{k}, v_{l}\right)}{B W_{\mathbf{v}}\left(v_{l}, v_{k}\right)}\right)^{\frac{1}{2}} \\
& =\sum_{\substack{k, l \in M \\
k \neq l}} B W_{\mathbf{v}}\left(v_{k}, v_{l}\right)=1,
\end{aligned}
$$

as desired.

4. Finally, we show that $b$ is a ratio scale. If $b^{\prime}$ is another such function, then when $i \neq m$,

$$
\begin{aligned}
b\left(v_{i}\right) & =\left(\frac{B W_{* \backslash v_{i}}\left(v_{i}, *_{m}\right)}{B W_{* \backslash v_{i}}\left(*_{m}, v_{i}\right)}\right)^{\frac{1}{2}} \\
& =\frac{b^{\prime}\left(v_{i}\right)}{b^{\prime}\left(*_{m}\right)} \\
& =a^{\prime} b^{\prime}\left(v_{i}\right)
\end{aligned}
$$

with $a^{\prime}=1 / b^{\prime}\left(*_{m}\right)$. 
Similarly, when $i=m$, there is $h \neq m$ with

$$
\begin{aligned}
b\left(v_{i}\right) & =\left(\frac{B W_{* \backslash v_{i}}\left(v_{i}, *_{h}\right)}{B W_{* \backslash v_{i}}\left(*_{h}, v_{i}\right)} \frac{B W_{*}\left(*_{h}, *_{m}\right)}{B W_{*}\left(*_{m}, *_{h}\right)}\right)^{\frac{1}{2}} \\
& =\frac{b^{\prime}\left(v_{i}\right)}{b^{\prime}\left(*_{h}\right)} \frac{b^{\prime}\left(*_{h}\right)}{b^{\prime}\left(*_{m}\right)} \\
& =\frac{b^{\prime}\left(v_{i}\right)}{b^{\prime}\left(*_{m}\right)} \\
& =a^{\prime} b^{\prime}\left(v_{i}\right)
\end{aligned}
$$

with $a^{\prime}=1 / b^{\prime}\left(*_{m}\right)$.

Thus, $b=a^{\prime} b^{\prime}$, so $b$ is a ratio scale.

\section{Acknowledgements}

This research has been supported by Australian Research Council Discovery Project Grants to the University of Technology, Sydney, for Louviere, Street and Marley (DP0343632) and for Louviere, Street, Wasi and Wang (DP0774142), by Natural Science and Engineering Research Council Discovery Grant 8124-98 to the University of Victoria for Marley. [ajm: We thank the referees for their careful study of the manuscript, and in particular for suggesting we expand the historical context of the problem and revisions to various technical details.]

\section{References}

Anderson, N. H. (1970). Functional measurement and psycho-physical judgment. Psychological Review, 77, 153-170.

Anderson, N. H. (1973). Comments on the articles of Hodges and of Schoenemann, Cafferty, and Rotton. Psychological Review, 80, 88-92.

Birnbaum, M. H. (1976). Intuitive numerical prediction. American Journal of Psychology, 89, 417-429.

Birnbaum, M. H., \& Stegner, S. E. (1979). Source credibility in social judgment: Bias, expertise, and the judge's point of view. Journal of Personality and Social Psychology, 37, 48-74. 
Birnbaum, M. H., \& Stegner, S. E. (1981). Measuring the importance of cues in judgment for individuals: Subjective theories of IQ as a function of heredity and environment. Journal of Experimental Social Psychology, 17, 159-182.

Birnbaum, M. H., \& Zimmermann, J. M. (1998). Buying and selling prices of investments: Configural weight model of interactions predicts violations of joint independence. Organizational Behavior and Human Decision Processes, 74, 145-187.

Birnbaum, M. H., Wong, R., \& Wong, L. (1976). Combining information from sources that vary in credibility. Memory $\mathscr{E}$ Cognition, 4, 330-336.

Coast, J., Salisbury, C., de Berker, D., Noble, A., Horrocks, S., Peters, T. J., \& Flynn, T.N. (2006). Preferences for aspects of a dermatology consultation. British Journal of Dermatology, 155, 387-392.

Finn, A. \& Louviere, J. J. (1992). Determining the appropriate response to evidence of public concern: The case of food safety. Journal of Public Policy and Marketing, 11, 1, 12-25.

Flynn, T. N., Louviere, J. J., Peters, T. J., \& Coast, J. (2006). Best-worst Scaling: What it can do for health care research and how to do it. Journal of Health Economics, 26, 171-189.

Green, P. E. (1974). On the design of choice experiments involving multifactor alternatives. Journal of Consumer Research, 1, 61-68.

Louviere, J. J. (1988). Analyzing Decision Making: Metric Conjoint Analysis. Sage University Papers Series Number 67. Newbury Park, CA: Sage Publications, Inc.

Louviere, J. J. (1994). Conjoint Analysis. In R. Bagozzi (Ed.), Advanced Marketing Research, Cambridge, MA: Blackwell Publishers.

Louviere, J. J. (2004). Complex statistical choice models: Are the assumptions true, and if not, what are the consequences? University of Technology, 
Sydney, CenSoC Working Paper No. 04-002.

Louviere, J. J., Carson, R., Ainslie, A., Cameron, T., DeShazo, J. R., Hensher, D. A., Marley, T., \& Street, D. (2002). Dissecting the random component. Marketing Letters (Special Issue on the UC Berkeley Invitational Choice Symposium), 13, 177-194.

Louviere, J. J., Hensher, D. A., \& Swait, J. D. (2003). Stated Choice Models. Cambridge: Cambridge University Press.

Louviere, J. J., \& Islam, T. (2008 in press). A comparison of importance weights and willingness-to-pay measures derived from choice-based conjoint, constant sum scales and best-worst scaling. Journal of Business Research..

Louviere, J. J., \& Woodworth, G. G. (1983) Design and Analysis of Simulated Consumer Choice or Allocation Experiments. Journal of Marketing Research, 20, 350-367.

Luce, R. D. (1959/2005). Individual Choice Behavior: A Theoretical Analysis. New York: John Wiley and Sons. Reprinted by Dover Publications, Mineola, NY (2005).

Luce, R. D. (1981). Axioms for the averaging and adding representations of functional measurement. Mathematical Social Sciences, 1, 139-144.

Luce, R. D., \& Tukey, J. W. (1969). Simultaneous conjoint measurement: a new type of fundamental measurement. Journal of Mathematical Psychology, $1,1-27$.

Marley, A. A. J. (1991). Context dependent probabilistic choice models based on measure of binary advantage. Mathematical Social Sciences, 1991, 21, 201-231.

Marley, A. A. J., \& Louviere, J. J. (2005). Some probabilistic models of best, worst, and best-worst choices. Journal of Mathematical Psychology, 49, 464-480. 
Marley, A. A. J. \& Luce, R. D. (2005). Independence properties vis-à-vis several utility representations. Theory and Decision 58, 77-143.

McIntosh, E., \& Louviere, J. J. (2002), Separating weight and scale value: an exploration of best-attribute scaling in health economics. Paper Presented to the Health Economists Study Group Meeting, Brunel University, July 3rd-5th, 2002.

Norman, K. L. (1976a). A solution for weights and scale values in functional measurement. Psychological Review, 83, 80-84.

Norman, K. L. (1976b). Weight and value in an information integration model: Subjective rating of job applicants. Organizational Behavior and Human Performance, 16, 193-204.

Norman, K. L. (1976c). Effects of feedback on the weights and subjective values in an information integration model. Organizational Behavior and Human Performance, 17, 367-387.

Norman, K. L. (1980). A case for the generalizability of attribute importance: the constant ratio rule of effects. Organizational Behavior and Human Performance, 25, 289-310.

Schoenemann, P. H., Cafferty, T., \& Rotton, J. (1973). A note on additive functional measurement. Psychological Review, 80, 85-87.

Shanteau, J. (1980). The concept of weight in judgment and decision making: A review and some unifying proposals. Report No. 228, July 1980, Centre for Research in Judgment and Policy, Institute of Behavioral Science, University of Colorado.

Street, A. P., \& Street, D. J. (1987). Combinatorics of Experimental Design. Oxford, UK: Oxford University Press.

Swait, J., \& Louviere, J. J. (1993). The role of the scale parameter in the estimation and comparison of multinomial logit models. Journal of Marketing 
Research, 30, 305-314. 